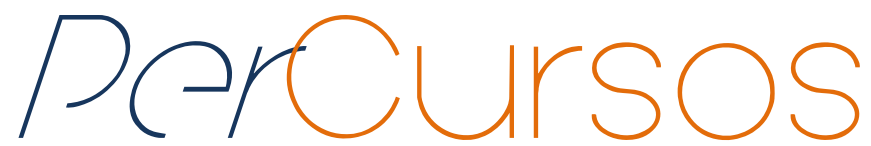

\title{
Emergencias étnicas/nacionales en una sala de Jardín de Infantes del Gran La Plata, Argentina
}

\begin{abstract}
Resumo
El Jardín de Infantes donde se realiza la investigación se encuentra ubicado en la región capital de la provincia de Buenos Aires, Argentina, donde se constata el asentamiento de poblaciones de distinta adscripción nacional y étnica. Se busca reconocer en el espacio áulico cuáles son los indicios de estas presencias y observarlas en el entramado de las socializaciones infantiles y escolares. En primer lugar describo la zona que contextualiza el establecimiento educativo para pensar la diversidad cultural en espacios escolares. Luego me pregunto si es posible observar la variabilidad étnica y nacional del Gran La Plata en una de las salas de la institución seleccionada. En este apartado analizo bajo qué modos aparece esta cuestión, con especial interés en el acceso a las voces de los niños y niñas. Desde la perspectiva etnográfica, tomo como fuente de análisis dos casos registrados en una sala integrada por las edades de 4 y 5 años. En último lugar, hago una breve referencia al acto escolar que conmemora el hecho histórico del 12 de octubre de 1492. La presencia de dos miembros Qom, en la ceremonia que se llevó a cabo en el Jardín de Infantes, amerita reflexionar sobre las maneras actuales de habitar el espacio escolar ante nuevas políticas que apelan al reconocimiento de la diversidad cultural como valor positivo y que tensionan los modos hegemónicos de la cotidianeidad de una escuela moderna.
\end{abstract}

Palavras-chave: Etnicidades y nacionalidades. Educación escolar de niñas y niños. Interculturalidad.
María Verónica Solari Paz

Licenciada en Antropología

(FCNYM/UNLP) y Mestranda em

Educación - FAHCE/UNLP. savecam@yahoo.com.ar

\section{Para citar este artigo:}

SOLARI PAZ, María Verónica. Emergencias étnicas/nacionales en una sala de Jardín de Infantes del Gran La Plata, Argentina . Revista PerCursos. Florianópolis, v. 14, n.26, jan./jun. 2013. p. 108 - 124.

DOI: $10.5965 / 1984724214262013108$

http://dx.doi.org/10.5965/1984724614262013108 


\section{Ethnic/national emergencies in a kindergarten classroom of the Gran La Plata,} Argentina

\begin{abstract}
The kindergarten in which we perform the research is located in the region of capital on the province of Buenos Aires, Argentina, where one observes the settlement of peoples of varied nationalities ant ethnic groups. We seek to recognise in the space of classroom what are the evidences of these presences and to observe them in the network of children and school socialisations. First, I describe the zone that contextualises the teaching establishment to think the cultural diversity in school spaces. Then, I ask if it is possible to observe the ethnic and national variety of the Gran La Plata in one of the classrooms of the chosen institution. I analyse in what way this question appears, with special attention to the access to the children's voices. In the ethnographic perspective, I take as source of the analysis two cases that happened in one classroom with children at the age of 4 and 5 years. Finally, I make a brief reference to the scholar act, which celebrates the historical fact of the 23th October 1492. The presence of two members Qom, in the ceremony that took place in the kindergarten, allows us to reflect on the contemporary ways if inhabiting the school space considering the new policies that appeal to the recognition of the cultural diversity as a positive value and that put a strain on the hegemonic ways of the day-byday of a modern school.
\end{abstract}

Keywords: Ethnicity and nationalities. School education of children. Interculturality.
Emergências étnicas/nacionais em uma sala de Jardim de Infância do Gran La Plata, Argentina

\section{Resumo}

O Jardim de Infância em que se realiza a pesquisa se encontra na região da capital da província de Buenos Aires, Argentina, onde se constata o assentamento de povoados de diversas nacionalidades e etnias. Busca-se reconhecer no espaço da sala de aula quais são os indícios destas presenças e observá-las na trama das socializações infantis e escolares. Em primeiro lugar, descrevo a zona que contextualiza o estabelecimento educativo para pensar a diversidade cultural em espaços escolares. Depois me pergunto se é possível observar a variedade étnica e nacional do Gran La Plata numa das salas da instituição selecionada. Então analiso de que modo aparece esta questão, com especial interesse no acesso às vozes das crianças. $\mathrm{Na}$ perspectiva etnográfica, tomo como fonte de análise dois casos registrados em uma sala integrada pelas idades de 4 e 5 anos. Em último lugar, faço uma breve referência ao ato escolar, que comemora o fato histórico de 12 de outubro de 1492. A presença de dois membros Qom, na cerimônia que aconteceu no Jardim de Infância, permite refletir sobre as maneiras atuais de habitar o espaço escolar ante novas políticas que apelam ao reconhecimento da diversidade cultural como valor positivo e que tensionam os modos hegemônicos da cotidianidade de uma escola moderna.

Palavras-chave: Etnicidades e nacionalidades. Educação escolar de crianças. Interculturalidade. 


\section{Introducción}

El Jardín de Infantes, público y provincial, donde se realiza la investigación se encuentra ubicado en la región capital de la provincia de Buenos Aires, Argentina. Es una de las provincias que mayor población reúne como lugar de destino de procesos migratorios. Me enfocaré en las construcciones sobre el Otro que se ponen en juego en la cotidianeidad escolar, a sabiendas de que el Jardín de Infantes en cuestión está localizado en una ciudad donde se constata el asentamiento de poblaciones de distinta adscripción provincial, nacional y étnica, datos tomados a partir de trabajo de campo y censales. Se busca reconocer en el espacio áulico cuáles son los indicios de estas presencias y observarlas en el entramado de las socializaciones infantiles y escolares.

Este trabajo consta de tres partes. En primer lugar describo la zona que contextualiza el Jardín de Infantes (en adelante J) y el fundamento de su elección para pensar la diversidad cultural en espacios escolares.

En el segundo apartado me pregunto si es posible observar la variabilidad étnica y nacional del Gran La Plata en una de las salas del Jl seleccionado. Bajo qué modos aparece esta cuestión y, especialmente, sobre la posibilidad producir conocimiento a partir de la perspectiva de niños y niñas ${ }^{1}$. Tomo como fuente de análisis dos casos registrados en una sala integrada por las edades de 4 y 5 años, de turno tarde, en el contexto de una etnografía escolar realizada en el año 2010. Como estrategia me propuse contemplar aquellas situaciones en que entablé diálogos con aquellos que inicialmente por sí mismos se acercaran a hablar o interactuar conmigo. Mi lugar era constante en la sala, siempre en la misma mesa.

En último lugar, hago una breve referencia al acto escolar del 12 de octubre, que tiene como invitados a una mujer y a un hombre, ambos qom, para ponerlo en relación con los casos ofrecidos. Esta articulación con el proceso de identificación étnica o

\footnotetext{
1Desde las investigaciones de Margaret Mead se acrecentaron las contribuciones a la comprensión e interpretación de formas diferentes de concebir y experimentar la niñez, lo que llevó hasta hoy la problematización sistemática de la categoría de infancia y de niñez. A finales del siglo XX, un movimiento con autonomía conceptual denominado sociología de la infancia fue desplegando una concepción de niño activo en la construcción de su propia vida social y de la sociedad misma. En esta línea se inscriben los estudios de Jens Qvortrup, William Corsaro, Chris Jenks, Allison James y Alan Prout.
} 
adscripción nacional atravesado por los debates educativos contemporáneos de diversidad, multiculturalidad e interculturalidad, permiten pensar de qué modo estos conceptos se corresponden con la agenda política y educativa en aspectos que hacen a lo “cultural” en mundos sociales cada vez más complejos, propios de los entramados urbanos contemporáneos.

\section{Cabalgando entre lo étnico y nacional en la trama escolar}

El interés por investigar las prácticas escolares en el nivel educativo inicial parte de poner en tensión el discurso oficial curricular que señala que el jardín de infantes debe responder a las características del contexto sociocultural en el que viven estos niños. En este sentido revisé las diferentes temporalidades, enunciados y narrativas de los grupos que hacen el aula del Jl urbano, de la localidad de Melchor Romero, región capital bonaerense.

Metodológicamente, procuramos conocer el espacio urbano antes de ingresar a esta institución. Este acercamiento nos llevó primeramente a recorrer el oeste de la ciudad de La Plata, donde registramos construcciones edilicias de alto poder adquisitivo muy cercanas a nucleamientos de viviendas de cartón, chapa o materiales premoldeados; con presencia activa de servicios públicos frente a la ausencia total de ellos; con calles asfaltadas y sendas que se forman por el intenso tránsito peatonal. Esa heterogeneidad se visualiza al localizar instituciones educativas y de salud públicas y privadas; establecimientos productivos, comerciales, artísticos, deportivos; presencia/ausencia de los servicios públicos (transporte, recolección de residuos, luz eléctrica, gas natural, cloacas, etc.); presencia y estado de fuentes de agua a cielo abierto; basurales, etc. La dinámica de este espacio está dada por la movilidad de su población (sub-alquileres transitorios de casas por cuestiones laborales, en fines de semanas largos, etc.).

La investigación antropológica en $\mathrm{Jl}$ públicos en jurisdicción de una de las delegaciones municipales del partido de La Plata nos confirma lo que veníamos observando respecto a la presencia compleja de procesos generados por los movimientos de grupos domésticos migrantes que luchan por lograr un espacio donde establecerse y 
organizar su existencia. En los últimos veinte años constatamos cambios sociodemográficos ocurridos en el oeste platense por el asentamiento de familias oriundas de diversas geografías; entre ellas, algunas que se adscriben como qom, quechuas, aymaras, kollas, gitanas (SOLARI PAZ; GARCÍA; CAPPANNINI, 2009). Dichos cambios no sólo producen transformaciones en el espacio territorial urbano sino en la organización de los servicios de abastecimiento de luz eléctrica, gas, agua, salud, educación escolar.

Malajovich (2006) describe la situación de los Jl en las últimas décadas en Argentina, en la que observa los efectos de la obligatoriedad para 5 años que establecía la Ley Federal de Educación de 1993, y que acentúa la huella de una desigualdad en las oportunidades de acceder a esta educación escolar en el transcurso de la infancia temprana. La educación sistemática se desarrolló con gran disparidad de la cobertura entre provincias, hacia adentro del nivel (entre el jardín maternal y el Iا) y entre edades de niños y niñas dentro del mismo Jl. Estas diferencias se encuentran combinadas a una notable desigualdad social ${ }^{2}$. La ley a la que hace referencia la autora no fue ajena a la forma en que el estado argentino asumió la gubernamentalidad en los '9o, bajo lo que se conoce como una "retirada del estado", esto es, una tercerización de servicios sociales claves, que a nivel micro involucró una redefinición de los sujetos gobernables, como en el caso de "pobres" y "subdesarrollados" que pasaron a ser "poblaciones vulnerables con capital social" (BRIONES, 2008, p. 11)

A falta de un Estado garante, muchos de los $\mathrm{J}$ corresponden a la gestión privada y en los segmentos más pobres a iniciativas de corte comunitario, los cuales recién fueron reconocidos oficialmente a partir de las sanciones de la ley nacional (2006) y provincial (2007). El Jl que tomo como referente empírico es uno provincial y público ${ }^{3}$, ubicado en el centro de la localidad de Melchor Romero; también existen otros dos establecimientos educativos: un JI privado religioso y uno público. Éste último también es provincial; en sus

\footnotetext{
2"la cobertura total y la oferta pública son escasas, en particular para los niños de 3 y 4 años si se las compara con los restantes niveles de enseñanza. También son las más injustas, ya que en el estrato más pobre, sólo 3 de cada 10 niños asisten a guarderías y jardines, mientras en el segmento de mayor poder adquisitivo la proporción sube a 9 de cada 10 niños". (Malajovich, 2006, p. 109).

3Se denomina así comúnmente a los jardines de infantes que corresponden al sistema de instrucción pública centralizado estatal en jurisdicción provincial.
} 
inicios, hace diez años, ocupó parte de las instalaciones municipales asistenciales con funciones de "guardería"; hace tres años, en el marco del presupuesto participativo, la comunidad vecinal votó por la edificación de una sede para dicha institución en el mismo predio. Si bien son tres los JI céntricos, dentro de un radio de seis cuadras, cabe indicar que en los últimos años el $\mathrm{Jl}$ de referencia en mi trabajo, ha reducido progresivamente con los años el servicio educativo a niños y niñas de tres años, en procura de abrir espacio para los que se inscriben con cuatro y cinco años de edad. Uno de los efectos de esta disposición la registré en la sala donde realicé mis observaciones; la misma era llamada por los docentes como "sala integrada" o "impura", por estar conformada por dos grupos de edades diferentes, de cuatro y cinco años, a diferencia de las salas "puras", conformadas por niños y niñas de un mismo grupo etáreo. En los documentos, como el Libro de Matrículas, la sala recibía la denominación de "sección múltiple”, respondiendo a la organización curricular de un grupo escolar formado por alumnos que cursan diferentes años de escolaridad ${ }^{4}$, en el mismo espacio, al mismo tiempo y con el mismo docente. Estos profundos cambios en la organización áulica e institucional se enmarcan en procesos macro-estructurales que exponen tres puntos de referencia problemática del JI en general; en forma separada y relacionados entre sí ocupan centralidad en la agenda del debate educativo (SVERDLICK; PAGANO; BORZESE, 2009): el "derecho a la educación”, “universalización de la educación infantil” y la “participación de la ciudadanía en la política pública".

En procura de profundizar en el cuestionamiento de las ausencias, de saberes, contenidos, referencias espacio-temporales, y producir conocimiento a partir de las emergencias (DE SOUSA SANTOS, 2006) que se revelan de la experiencia misma y contribuir a hacerlas presentes, la pregunta que guía esta presentación es si es posible observar la diversidad cultural que acusa el poblamiento de los últimos años en la sala de Jl, y bajo qué o cuáles condiciones hacen posible su emergencia en un contexto donde históricamente el Otro ha sido excluido y hasta despreciado; me habilito a preguntarme

4Glosario, realizado por el Departamento de Análisis de la Información. Dirección de Información y Estadística de la Dirección General de Cultura y Educación de la Provincia de Buenos Aires. (DGCYE, 2006: 41) 
sobre la posición de unos que han sido excluidos en detrimento de otros. A partir del S. XIX la escuela se expandió como forma educativa dominante a nivel mundial; “han servido para inculcar habilidades, subjetividades, y disciplinas que refuerzan el estado-nación moderno" (Durkheim 1956 en LEVINSON Y HOLLAND, 1996). La escuela se ha consolidado como el lugar privilegiado para adquirir conocimientos y habilidades necesarios para desenvolverse y participar como ciudadano; pero el saber escolar que transmite es diferencial, sujeta/posiciona al individuo en un modo de producción global dominante, inscripto en una nación-estado y en un entramado de relaciones desiguales.

Esta desigualdad ha sido categorizada en el campo de las ciencias sociales tradicionalmente en cuanto a variables que hacen al sujeto moderno. En un indiscutido lugar se encuentra la clase (dominantes/populares; alta/media/baja). Otra variable es el género: de la clásica descripción que corresponde al sexo al nacer (femenino/masculino tal como se presentan los datos en los documentos escolares) a perspectivas actuales que problematizan la desigualdad de acuerdo a estados de identidad de género (mujer/varón/ $\mathrm{LGBT}^{5}$ ) y/o su orientación sexual (heterosexual/homosexual). La edad es una de las fuentes de comparación que en el campo educativo es central a la hora de diseñar los espacios institucionales: las edades de todos los individuos en su escolarización están agrupadas en etapas escolares, cada una dividida por años progresivos de escolarización. Una de las primeras relaciones de poder que aprendemos cuando nacemos es que quienes mandan son los más grandes, o los que estaban antes; a esto lo agrava la falta de correspondencia entre la edad cronológica y la de escolaridad.

En este trabajo me ocuparé centralmente de la nacionalidad junto a otro componente, la variable étnica; esta última, invisibilizada en los registros escolares bajo la categoría de nacionalidad hace pensar en este solapamiento como el binomio una nación/una etnia construido en nuestro país desde el siglo XIX. Los datos que figuran en los documentos escolares corresponden a la nacionalidad, tanto la de los padres como la de los alumnos; quedan asentadas en la documentación oficial del establecimiento para ser remitidas, ahora digitalmente, al banco de datos provincial y nacional. Fuera de las

5 L: Lesbianas; G: Gays; B: Bisexuales y T: Transgénero 
fuentes escritas me propuse indagar este tema en la interacción en la sala. En años anteriores, vi como en el aula de primaria es común la evidencia a través de los insultos entre compañeros, o en la explicación que dan algunos docentes respecto al desempeño de algunos de sus alumnos/as. Durante el trabajo de campo en el Jl, apareció de la voz de un niño una referencia a la nacionalidad, caso que analizaré a continuación.

\section{Emergencias Étnicas Orales En La Sala}

A través de un trabajo conjunto entre maestras y directivos, propusieron para el año 2009 un proyecto en el que participaran al mismo tiempo todas las salas y que culminara en una actividad común. Según los informantes, debía transmitir contenidos que se encuentran en el currículum vigente pero al mismo tiempo decidieron que debía tener en cuenta a la población étnicamente diversa que asiste, a la que conocen a través de las entrevistas iniciales que hacen a los padres/madres o tutores de los alumnos/as. Este proyecto se llamó "El vivero" y fue replicado al año siguiente, en el mes de septiembre, momento al que pertenecen todos los fragmentos citados en este trabajo. Un antecedente de este proyecto institucional curricular fue pensar en la huerta; la idea provino de una maestra oriunda del noroeste argentino, que ya no está en el Jl, pero a cuya idea adhirieron el resto de sus colegas. Su proyecto se inscribió en la huerta como objeto escolar, un modo que integra los aspectos de producción agrícola local y la cosmovisión que toma en cuenta la unidad con la tierra, bajo la figura de la Pachamama.

Bajo este marco institucional, cada maestra diseñó para su sala un plan de actividades que según ésta podía demandar una, dos o tres semanas, con culminación el 21 de septiembre, fiesta escolar del "Día de la primavera". En la sala ${ }^{6}$ donde desarrollé mi trabajo de campo, la actividad central ceñida a este proyecto fue el proceso de germinación. En días anteriores fue acompañado por la descripción del proceso de

\footnotetext{
6 La sala agrupa 18 niños (10 de ellos egresan en 2010) y 12 niñas (donde sólo 2 egresan ese año). El resto de niños y niñas egresarán del $\mathrm{JI}$ en 2011. Las clases se desarrollan en el turno tarde (13hs p.m. hasta 17hs p.m.). Hasta mediados de mayo tuvieron a una maestra que luego renunció al cargo por obtener la titularidad como directora en otro ji de la misma localidad. Fue reemplazada por una maestra que tuvo el grupo a cargo como titular hasta fin del año lectivo.
} 
crecimiento y partes de la planta a través de láminas con gráficos y nombres. La germinación fue presentada tal como se hace el procedimiento en la escuela primaria. A continuación se muestra un fragmento de lo registrado en esos días:

Las semillas utilizadas en la germinación primero fueron porotos, luego semillas de naranja, finalmente de maíz. Al observar estas últimas, Kevin se acerca a contarme que "eso se hace chiquito" (señala a la semilla de maíz), todo me lo decía al oído, evitando que los demás escuchen: "yo lo sé, porque lo vi en Bolivia". Ah, sí?, dije curiosa, y esperando que cuente más. "Se hace chiquitito (y apretaba sus deditos índice y pulgar entre sí)", y entusiasmado continuó explicando cómo se hace la molienda del maíz; ya no en secreto y gesticulando con sus brazos hablaba de unas ruedas de piedra muy grandes, que se movían y que aplastaban las semillas, dejándolas muy chiquitas. ¿Y qué se hace con eso? Para qué las dejaban así? pregunté. "No sé" dijo y se volvió a su asiento de modo pensativo; luego volvió y agregó "eso es para comer".

En este breve diálogo el niño me comunica su conocimiento sobre el objeto presentado por la maestra, además del proceso de molienda del maíz, es decir, de su tratamiento para transformarlo en otro producto comestible. Esta traslación que hace el niño del objeto en un esquema de experimentación artificial al objeto en un contexto de producción, trae a colación la reflexión sobre que no importa cuán definida esté en su mundo social inmediato la persona entendida (Kevin en este caso); al parecer, las escuelas persisten en no tener en cuenta las habilidades y sensibilidades que cuentan como indicadores de 'sabiduría' e inteligencia en el hogar y la localidad inmediata (LEVINSON Y HOLLAND, 1996). Queda para otro trabajo la profundización teórica sobre cómo la escuela interpone históricamente una misión educacional de proporciones extralocales, convirtiendo en un proceso mundial la transmisión de los mismos contenidos, la universalización y simplificación en este caso favorecidos por la abstracción del reino vegetal, reducido a un esquema de planta con tallo erguido, flor en la punta, dos hojas, una a cada lado del tallo y raíces por debajo de la tierra y que se reproducen por semilla.

La referencia que hace el niño sobre una situación vivida años antes en el país vecino da indicios de que su trayectoria posiblemente estuviera asociada a procesos 
migratorios ${ }^{7}$. Pero el contexto comunicacional brinda mucho más datos que ese. La forma del secreto nos abre las puertas para explorar la valoración que él realiza respecto de cómo es considerada esa nacionalidad en este lugar. ¿Por qué dice en secreto un saber que, claramente, él pondera?. Me detengo en la base de la acción, el secreto, como una forma decidida por él y que requiere de su capacidad para la significación. Decide no decir abiertamente dónde y cómo lo aprendió, en una evaluación de lo que esas palabras pueden connotar en este aquí y ahora escolar.

El sometimiento a un silenciamiento público del fragmento anterior se opone a lo que vivencié a principio de año en mi primer día de trabajo de campo en la misma sala. Era el mes de marzo y la jornada escolar era de sólo dos horas. En el momento de espera para recibir a los padres que los vendrían a retirar del Jl, la maestra invita a todos a cantar lo que quieran. Los que se animaron fueron los niños; al principio uno cantó la canción al Jl que cantan todos cuando inicia la jornada diaria con el izamiento a la bandera. Luego la maestra pidió otras canciones, las que les gustara cantar en sus casas o con amiguitos, y así se abrió paso a canciones en guaraní, que poco a poco, algunos se incorporaron al unísono. Luego uno cantó Manuelita de María Elena Walsh y la maestra preguntó si sabían qué era Pehuajó; mi respuesta obvia se vio atropellada por la sorpresa. Supuse que la pregunta era sobre si sabían de un lugar geográfico como es la ciudad de Pehuajó; algunos respondieron lo que la maestra quería saber: respondieron con su traducción del guaraní, significados que referían al agua. Finalmente un niño quiso cantar el Elefante Trompita, a la que se plegaron a cantar todos. La convocatoria a expresar sus "gustos" a través del canto, reveló una valorización que a grandes rasgos se puede agrupar en canciones en una lengua a que no se tiene en cuenta como otra lengua hablada en esta provincia. Ante la canción del Jl, que si consideramos que es el mes de marzo y el primer año de escolarización, resultan atractivas las observaciones de Brubaker y Cooper (2001) respecto al poder del Estado moderno como uno de los agentes más importantes de la identificación y la categorización. Las otras dos son canciones infantiles muy populares en

\footnotetext{
7En diciembre, en contexto de la fiesta de egresados, le conté a su mamá lo ocurrido. Confirmó la nacionalidad boliviana de ambos; la madre estaba muy conmovida por el hecho de que Kevin recordara lo que vivió hasta sus dos años de edad en su país de origen.
} 
Argentina, que ingresaron al mercado de la música a mediados de siglo XX: el Elefante

Trompita en 1947 y Manuelita en 1962 (y continúan hasta la actualidad sus reediciones).

Tanto la situación comentada de la germinación como esta última se corresponden a dos escenas con actores diferentes en el mismo espacio áulico. En los dos casos el alumnado es el mismo, la investigadora también; pero en el segundo caso la maestra titular estuvo a cargo hasta junio; luego asumió esa titularidad la maestra involucrada en el primer fragmento. Es notable la variación del espacio de apertura que generan las maestras para que los niños/as desplieguen sus saberes. Inquieta saber cuáles son las consecuencias que traen aparejadas estos dos modos de relación que establecen las maestras, sea en la diferencial relación entre el saber escolar y saberes otros, sea en la relación entre adulta y niños/as, o entre maestra y alumnas/os. Diez (2011) estudia las biografías y trayectorias de los niños/as migrantes en vinculación con los procesos de subalternización de sus repertorios sociohistóricos y experiencias vitales. Incluye la escuela como una experiencia vital en la que los sujetos revelan ciertas posiciones asumidas en torno a sus identificaciones, que en relación al contexto inmediato ejercen su derecho a hacerlo público o no.

Esto abre un camino para problematizar, según lo planteado hasta ahora, la pertinencia y alcance de los estudios que a fin de entender la heterogeneidad y desigualdad de los sujetos en instituciones escolares, que versan desde la importancia de analizar la relación pedagógica, los contextos sociales de los niños como espejos de identidad, el lenguaje de los cuerpos en el espacio escolar, las perspectivas que ponderan las voces de los niños/as en la interpretación.

\section{Vivencias Y Escritos Sobre La Otredad En El Jardín De Infantes}

Las políticas globales en torno a la diversidad ingresan en el plano educativo de nuestro país en la década 1990 con la Ley Federal de Educación. Sedimentadas por una ideología de respeto, un hito en América como lo es la Carta de Derechos y Libertades en Canadá (1975) y la difusión desde EEUU del multiculturalismo. Esta corriente de pensamiento apunta a un reconocimiento público dentro de un estado-nación de las 
diferencias culturales, bajo una mecánica de tolerancia y convivencia. Los cambios en las constituciones en países de América del Sur reconociéndose como pluriculturales ocurren en la década de 1990, simultáneamente con el incremento de la pobreza y el desempleo.

En nuestro país toma fuerza el concepto "educación intercultural”, inscripto en una política estatal que empieza en la última década del siglo XX e inicios del XXI en paralelo a otros países de América Latina, como propuesta a los problemas que plantea la educación escolar de los que se adscriben como pertenecientes a los pueblos originarios, y en respuesta a un fortalecimiento de las organizaciones indígenas y a un derecho internacional y nacional que avala estos procesos reivindicativos.

En el partido de La Plata tiene una presencia activa la Asociación Civil Ntaunaq Nam Qom y otras comunidades qom, que si bien no constituyen una organización civil, forman parte del colectivo de los "movimientos sociales que apelan a una variedad de reformas de los derechos territoriales y políticos, de la autonomía cultural y, en algunos casos, simplemente del derecho a la vida" (WADE, 2000). El Jl, como vimos anteriormente, no es ajeno al entramado político urbano. En el acto escolar, dos miembros Qom se presentaron con previa solicitud y anuencia de la directora y supervisora en la conmemoración del 12 de octubre, fiesta calendárica denominada en nuestro país como "Día de la Raza”: la fecha remite a la llegada de Colón a este continente, mientras que su denominación se ubica en la década de los años 1930s en alusión a la raza como hispanidad, asume la carga significante de una etnia- una nación. El 12 de octubre escolar representa una huella de subordinación histórica: "huella en el cuerpo del paso de una historia otrificadora que construyó "raza" para constituir "Europa" como idea epistémica, económica, tecnológica y jurídico-moral que distribuye valor y significado en nuestro mundo" (SEGATO, 2007, p. 23). Esta llamada experiencia otrificadora por la autora, con el bagaje colonizador que lo contextualiza, es combatida en la conmemoración del V Centenario. Movimientos sociales indígenas apelan, discuten su presencia en el presente, lucha que llevó a que gobiernos nacionales hayan adoptado medidas políticas, tal el caso de la reforma constitucional de nuestro país en 1994, que reconoce la composición multiétnica que se aleja discursivamente de un nacionalismo basado en la ciudadanía homogénea. 
Los qom en el acto escolar contaron los modos de vida en el noreste argentino, desde los tradicionales hasta la acuciante realidad debido a la reducción de sus recursos que hacían imposible vivenciarlos. Una de las actividades predominantes de los qom en las ciudades es la producción y venta de artesanías. En su relato los qom respondieron preguntas de las maestras respecto a su pasado y a su actualidad en las ciudades, y a los niños/as en relación con las figuras de arcilla y qué representaban. Luego del acto, estas figuras junto a accesorios de indumentaria con semillas estuvieron a la venta. La jornada del acto terminó a media mañana, luego los invitados partieron a otro Jl de la misma localidad. Supe por ellos que varios de sus compañeros se organizaron para ir a otros centros educativos, con mayor preponderancia de JI que escuelas primarias.

Es interesante la contribución de Czarni en su estudio de la membresía como concepción mediadora para el análisis de la relación entre pueblos indígenas y escolaridad, al dilucidar que "el análisis de la escolaridad desde un concepto amplio de “diferencias" y “exclusión” -cultural, étnica, racial, lingüística, género, etcétera- ha impulsado el cambio de lente para leer el acto escolar alrededor de estos grupos; diversos trabajos ya no plantean lo que la escuela "hace" con las comunidades y los actores subordinados sino lo que éstos hacen con la escuela" (CZARNY, 2007, p. 927).

Siguiendo este planteo, propongo entender la entrada de los qom en el $\mathrm{J}$ como una negociación que se establece entre dos miembros de una etnia conocida en la zona y algunos agentes escolares que los incorporaron en el espacio escolar, contemporáneamente a la implementación de lineamientos que proponen diálogos interculturales como mecanismo para transitar las diferencias. La inteculturalidad, a diferencia del multiculturalismo, se propone como una transformación de la forma de ver, que no está ajena a las relaciones de poder y los modos de producción.

El uso del término interculturalidad como perspectiva, método, acto, o como subjetividad predispuesta a entender el mundo a través de los ojos del otro, da cuenta de la polisemia del mismo. Su institucionalización como contenido curricular en el campo educativo adquirió la forma en la Provincia de Buenos Aires de modalidad transversal, denominada "Modalidad intercultural”. A nivel nacional se implementó el programa de Educación Intercultural Bilingüe. Estos esfuerzos del Estado por responder y dar lugar a 
estrategias que apunten a la contextualización del currículum a los modos de vida y a la lengua indígena se ponen de manifiesto en prácticas discursivas que generan apropiaciones diferenciales en la vida cotidiana. Esta finalidad algunos la inscriben como dispositivos de la modernidad que ligan pobreza y rendimiento (GWIRTZ et al, 2007), otros como un derecho a la ciudadanía a debatir la educación.

\section{Conclusiones}

Este texto proporciona indicios de identificaciones étnico-nacionales, entre otros procesos de identificación infantiles que ocurren en la diferencia y desigualdad que caracteriza a esta institución educativa. Las agencias infantiles con edades de cuatro y cinco años pusieron de manifiesto el guaraní como lengua hablada, el relato de una experiencia en Bolivia con emociones, conocimientos adquiridos en esa experiencia, la subalternidad asumida en el espacio escolar. Estas emergencias están en tensión con su procesamiento social en clave de normalidad/ anormalidad, inclusión/ exclusión, nacional/ extranjero, castellano/ otras lenguas, que clasifica y jerarquiza la escuela. Abordar la subalternidad de la voz de los niños y niñas en tanto alumnos sumado a la subalternidad que tiene una lengua-otra, la etnia indígena, la nacionalidad de países limítrofes del noroeste argentino, la pertenencia a una familia migrante en la escuela, constituye en sí un problema. Dos lógicas diferentes lo imbrican, expresados en dos derechos reconocidos en la carta de los derechos de niños, niñas y adolescentes, a saber:

- niño/a con derecho a la educación (la educación escolar es extralocal, universal, atemporal)

- derecho a la identidad (temporal y espacialmente localizado).

En los materiales que se analizaron en este trabajo, las posibilidades del segundo derecho dependen de la agencia de los sujetos, pero por sobre todo de las condiciones que posibilita el contexto escolar. $Y$ estas condiciones no son fortuitas, requieren de la profundización de estos temas, y que sean visibles en la agenda de los temas a discutir para ser llevados al aula. Vale agregar que las categorías de multiculturalidad e 
interculturalidad no aparecen en el lenguaje oral y en las producciones escritas de los docentes, sino en el lenguaje escrito de los documentos oficiales.

La incorporación de las voces de los niños y su corporalidad se suma a las interpretaciones que forman parte de este análisis. Metodológicamente trabajé sobre la "voz" de aquellos que querían hablar y/o ser escuchados ¿Cuál es la voz de los que no dijeron, la "no voz"? ¿Son acaso voces que siempre quedan postergadas o silenciadas en el ámbito público? ¿es el caso de las niñas, de los que no hablan con nadie, de los que sienten que su voz no tiene peso? Si sostenemos que las voces de la infancia suman a las interpretaciones que tenemos del mundo social, queda pendiente problematizar el cómo accedemos a todas las voces en la sala.

Es importante señalar la importancia que están cobrando los estudios sobre infancias dentro del campo de las ciencias sociales para entender procesos macroestructurales (CARLI, 2006), en el campo educativo (ROCKWELL, 2001; JOCILES RUBIO, 2006), en la relación escuela-comunidad (CZARNY, 2007; NOVARO, 2011), el discurso de los niños en las narrativas antropológicas (MILSTEIN, 2008), y la contribución de los niños a la sociedad en general. Cobran reconocimiento y validez las voces de los niños y niñas, lo que necesariamente demanda una problematización y explicitación de los presupuestos sobre su estatuto en la distribución de poder en el entramado de relaciones con los adultos, con un docente, conmigo (ahí y al mismo tiempo en una contemporaneidad distinta como es en el proceso de investigación).

\section{Bibliografía}

BRIONES, C. Formaciones de alteridad: contextos globales, procesos nacionales y provinciales. En: BRIONES, C. Cartografías Argentinas: políticas indigenistas y formaciones provinciales de alteridad. 1a. ed. Buenos Aires: Antropofagia, 2008. Cap. 1, p. 9-36.

BRUBAKER, R.; COOPER, F. Más allá de "identidad". Apuntes de investigación - CECYP, Buenos Aires, v. 5, n. 7, p. 30-67, 2001. 
CARLI, S. Niñez, pedagogía y política: transformaciones de los discursos acerca de la infancia en la historia de la educación argentina entre 1880 y 1955. 1a. ed. Buenos Aires: Miño y Dávila, 2002.

CZARNY KRISCHKAUTZKY, G. Pasar por la escuela. Metáfora que guarda distitnas caras para abordar la relación comunidades indígenas y escolaridad. Revista Mexicana de Investigación Educativa., México D. F., v. 12, n. 34, p. 921-950, Julio-Septiembre 2007.

DE SOUSA SANTOS, B. Capítulo I. La Sociología de las Ausencias y la Sociología de las Emergencias: para una ecología de saberes. En: DE SOUSA SANTOS, B. Renovar la teoría crítica y reinventar la emancipación social (encuentros en Buenos Aires). 1a. ed. Buenos Aires: Consejo Latinoamericano de Ciencias Sociales, 2006. Cap. 1, p. 31-41.

DIEZ, M. L. Biografías no autorizadas en el espacio escolar. Reflexiones en torno a ser migrante en la escuela. En: NOVARO, G. La interculturalidad en debate. Experiencias formativas y procesos de identificación en niños indígenas y migrantes. 1a. ed. Buenos Aires: Editorial Biblos, 2011. Cap. 9, p. 153-177.

JOCILES RUBIO, M. I. Diferencias culturales en la educación. Apuntes para la investigación y la intervención. Gazeta de Antropología. Universidad Complutense de Madrid, Madrid, n. 22 - Artículo 22, agosto 2006. Disponible en:

http://www.ugr.es/ pwlac/G22_27Marialsabel_Jociles_Rubio.html. Fecha de acceso: 22 de jun. 2012.

LEVINSON, B.; HOLLAND, D. La producción cultural de la persona educada: una introducción. En: LEVINSON, B.; FOLEY, D.; HOLLAND, D. The cultural production of the educated person: critical ethnographies of schooling and local practice. 1. ed. Albany: State University of New York Press, 1996. Cap. 1, p. 1-25.(traducción de Laura Cerletti).

MALAJOVICH, A. Experiencias y reflexiones sobre la educación inicial: una mirada latinoamericana. 1a. ed. Buenos Aires: Siglo XXI Editores Argentina, 2006.

MILSTEIN, D. Infancias y política en la antropología de la educación argentina. Avá, Posadas, n. 15, p. 307-314, Julio 2009. 
ROCKWELL, E. Caminos y Rumbos de la Investigación Etnográfica en América Latina.

Cuadernos de Antropología Social. Facultad de Filosofía y Letras, UBA, Buenos Aires, n. 13, p. 53-64, 2001.

SEGATO, R. Introducción: políticas de la identidad, diferencia y formaciones nacionales de alteridad. En: SEGATO, R. La Nación y sus Otros: raza, etnicidad y diversidad religiosa en tiempos de políticas de la identidad. 1a. ed. Buenos Aires: Prometeo Libros, 2007. p. 15-36.

SOLARI PAZ, VERÓNICA; GARCÍA, STELLA MARIS; CAPPANNINI, MARIEL. Las máscaras de la alteridad en escuelas platenses. Perspectiva antropológica. Revista de Educación de la Universidad Nacional de Rosario, Rosario, v. 5, n. 4, p. 237-258, Diciembre 2009.

SVERDLICK, INGRID; PAGANO, ANA; BORZESE, CECILIA. La situación de la primera infancia en la Provincia de Buenos Aires. La acción de las organizaciones y movimientos sociales. Universidad Pedagógica de la Pcia. de Bs. As. La Plata, p. 1-63. 2009. Disponible en: http://unipe.edu.ar/wp-content/uploads/2011/11/Ingrid-Sverdlick.pdf. Fecha de acceso: 10 de nov. 2012.

WADE, P. Los negros y los indígenas en la nación estado posmoderna. En: WADE, P. Raza y etnicidad en Latinoamérica. 1a. ed. Quito: Ediciones ABYA-YALA, 2000. Cap. 5, p. 97-113.

Recebido em: 17/03/2013 Aprovado em: 16/05/2013

Universidade do Estado de Santa Catarina - UDESC Centro de Ciências Humanas e da Educação - FAED

Revista PerCursos Volume 14 - Número 26 - Ano 2013 revistapercursos@gmail.com 